\title{
Analysis of Factors Affecting Positron Emission Mammography (PEM) Image Formation
}

\author{
Mark F. Smith, Member, IEEE, Stan Majewski, Andrew G. Weisenberger, Douglas A. Kieper, \\ Raymond R. Raylman, and Timothy G. Turkington, Member, IEEE
}

\begin{abstract}
Image reconstruction for positron emission mammography (PEM) with the breast positioned between two parallel, planar detectors is usually performed by backprojection to image planes. Three important factors affecting PEM image reconstruction by backprojection are investigated: 1) image uniformity (flood) corrections, 2) image sampling (pixel size) and 3) count allocation methods. An analytic expression for uniformity correction is developed that incorporates factors for spatial-dependent detector sensitivity and geometric effects from acceptance angle limits on coincidence events. There is good agreement between experimental floods from a PEM system with a pixellated detector and numerical simulations. The analytic uniformity corrections are successfully applied to image reconstruction of compressed breast phantoms and reduce the necessity for flood scans at different image planes. Experimental and simulated compressed breast phantom studies show that lesion contrast is improved when the image pixel size is half of, rather than equal to, the detector pixel size, though this occurs at the expense of some additional image noise. In PEM reconstruction counts usually are allocated to the pixel in the image plane intersected by the line of response (LOR) between the centers of the detection pixels. An alternate count allocation method is investigated that distributes counts to image pixels in proportion to the area of the tube of response (TOR) connecting the detection pixels that they overlay in the image plane. This TOR method eliminates some image artifacts that occur with the LOR method and increases tumor signal-to-noise ratios at the expense of a slight decrease in tumor contrast. Analysis of image uniformity, image sampling and count allocation methods in PEM image reconstruction points to ways of improving image formation. Further work is required to optimize image reconstruction parameters for particular detection or quantitation tasks.
\end{abstract}

\section{INTRODUCTION}

F unctional imaging of the breast with F-18 fluorodeoxyglucose (FDG) has the capability to differentiate metabolically active tumors and is being investigated for the detection, staging and treatment of breast cancer [1-4].

M. F. Smith (telephone 757-269-5539, e-mail: mfsmith@jlab.org), S. Majewski, A. G. Weisenberger, and D. A. Kieper are with the Thomas Jefferson National Accelerator Facility, Newport News, VA 23606 USA.

R. R. Raylman is with the Department of Radiology, Robert C. Byrd Health Sciences Center, West Virginia University, Morgantown, WV 26506 USA.

T. G. Turkington is with the Department of Radiology, Box 3949, Duke University Medical Center, Durham, NC 27710 USA.

The Southeastern Universities Research Association (SURA) operates the Thomas Jefferson National Accelerator Facility for the United States Department of Energy under contract DE-AC05-84ER40150.
Clinical breast imaging with FDG has been performed with conventional positron emission tomography (PET) scanners as well as with dedicated positron emission mammography (PEM) devices $[5,6]$. There have been many proposed designs for PEM detectors, which have the potential for improved sensitivity and spatial resolution compared with conventional PET scanners [5-17]. Initial clinical results have been reported only for the dual planar detector configuration, however $[5,6,11]$.

For a PEM device with the breast positioned between two static planar detectors, image reconstruction is usually performed by backprojection along the lines of response (LORs) to the desired image planes [18]. A lesion is focused when an image is formed in the plane in which it is located, while out-of-plane activity appears blurred. In this contribution three important factors affecting PEM image formation by the backprojection method are investigated: image uniformity (flood) corrections, image sampling (pixel size) and count allocation methods.

Image uniformity is dependent on spatially dependent geometric and detector sensitivity factors. An analytic expression for reconstructed flood images will be compared with experimentally measured flood images and will be used for uniformity corrections.

The ability to resolve small objects in PEM images is affected by the reconstructed pixel size. The appropriate image pixel size is dependent in part on the detector pixel size and on the raypath density of the LORs. Image reconstructions with pixel sizes equal to the detector pixel size and smaller than the detector pixel size will be examined.

Image reconstruction is often performed with a nearestneighbor approximation for allocating counts to image pixels. Counts for a pair of detection pixels on opposite detector heads are allocated to the image pixel that the LOR between the centers of the detector pixels intersects in the image plane. This count allocation method is compared with an alternate method of modeling the physics of data acquisition, in which annihilation events are modeled as occurring anywhere within the tube of response (TOR) connecting the pair of detector pixels. Counts are allocated to image pixels in proportion to the area of the TOR that they overlay in the image plane.

These factors affecting image formation will be investigated using mathematically simulated and experimentally acquired PEM acquisitions of compressed breast phantoms. 


\section{METHODS}

\section{A. Image Uniformity}

The reconstructed image of a sheet source of unit activity in plane $z$ parallel to the detector surfaces can be written

$$
\begin{aligned}
F(x, z)= & {\left[\int_{0}^{\theta_{\max }} \sin \theta d \theta \int_{0}^{2 \pi} d \phi D_{A}\left(x_{A}(x, z, \theta, \phi)\right)\right.} \\
& \left.D_{B}\left(x_{B}(x, z, \theta, \phi)\right) G(x, z, \theta, \phi)\right] /\left[2 \pi\left(1-\cos \theta_{\max }\right)\right]
\end{aligned}
$$

where $x$ is a location in plane $z$ and $\theta_{\max }$ is the maximum angle from normal incidence of accepted events contributing to the reconstructed image (Figure 1). The points $x_{A}$ and $x_{B}$ are the locations that the annihilation photons strike detectors $\mathrm{A}$ and $\mathrm{B}$, and $D_{A}$ and $D_{B}$ are the detector sensitivity functions. The geometric sensitivity function $G(x, z, \theta, \phi)$ is one if the paths of both annihilation photons are in the field of view of the detectors and zero otherwise. The acceptance angle $\theta_{\max }$ has a major effect on image uniformity through its effect on geometric sensitivity [14].

Experimental flood images can be compared with simulated images that incorporate the geometric sensitivity function and assume uniform detector response. If these images are in good agreement, then the effect of detector nonuniformity is small compared to the geometric factor. In this case it may be feasible to calculate flood images for uniformity corrections and avoid excessive flood measurements.

Experimental flood measurements were acquired on a dual detector PEM system with a $29 \times 29$ crystal array of $3.0 \times$ $3.0 \times 10 \mathrm{~mm}^{3}$ LGSO crystals. The crystals were wrapped with white Teflon tape, which resulted in a $3.3 \mathrm{~mm}$ crystal pitch in the detector array. The detector heads were positioned $18 \mathrm{~cm}$ apart and an F-18 flood source in a $15 \times 15 \times 2.5 \mathrm{~cm}^{3}$ box phantom was positioned $11.5 \mathrm{~cm}$ from one of the detector heads. This mimics the position of a breast when PEM detectors are positioned above the compression paddle and below the film tray of an $\mathrm{x}$-ray mammography system. Experimental flood images were reconstructed with different acceptance angles and compared with numerical simulations. The energy window for image reconstruction was $420-650$ $\mathrm{keV}$.

For the geometry of the above experimental acquisition, flood images were simulated using equation (1) with uniform detector sensitivity functions. The ratios of the experimental images to the simulated images were formed.

Calculated image uniformity corrections also were evaluated for an experimental breast phantom acquisition using a pixellated GSO detector [14]. The detector heads were each $10 \mathrm{~cm} \times 10 \mathrm{~cm}$ with $30 \times 30$ crystal arrays. Each crystal was $3.1 \times 3.1 \times 10 \mathrm{~mm}^{3}$ and wrapped in white Teflon tape to improve reflectivity. The center-to-center crystal pitch in the detector was $3.3 \mathrm{~mm}$.

The experimental acquisition was performed with F-18 in a $4 \mathrm{~cm}$ thick compressed breast phantom. Spherical lesions were $6,9,12$ and $15 \mathrm{~mm}$ diameter The activity concentration in the turnors was $0.55 \mathrm{microCi} / \mathrm{ml}$ and $0.065 \mathrm{microCi} / \mathrm{ml}$ in the background, for a tumor:background concentration ratio of $8.5: 1$ and total phantom activity of 28 microCi. The detectors were $20 \mathrm{~cm}$ apart and the breast phantom was placed midway between the detectors. The phantom was imaged for 2 minutes and the energy window for image reconstruction was 400-700 keV. Images were reconstructed with and without uniformity corrections for different acceptance angles. The signal-to-noise ratio was computed for the visible tumors using the formula

$$
S N=(T-B) / \sigma(B)
$$

where $T$ is the mean in a region of interest (ROI) in the tumor center, $B$ is the mean in an adjacent background ROI, and $\sigma(B)$ is the standard deviation in the background $\mathrm{ROI}$.

\section{B. Image Sampling}

Raypath density diagrams for a dual planar detector PEM system show that the raypaths sample the image space more finely than the detector pixel size (Figure 2). In the plane halfway between the detectors the raypath spacing is equal to half of the detector pixel size, suggesting that image pixel sizes smaller than the detector pixel sizes may be advantageous. A similar principle is adopted in conventional PET to form transaxial slices from cross-plane coincidence events.

To evaluate the effect of image pixel size, numerical PEM simulations of a compressed breast with tumors were made. The breast thickness was $6 \mathrm{~cm}$, the tumor diameters were 6 , 8,10 and $12 \mathrm{~mm}$ and the tumor:background activity concentration ratio was $8: 1$. The detector separation was 18 $\mathrm{cm}$ and the tumors were in a plane $11.5 \mathrm{~cm}$ from one of the detectors. Noise-free coincidence data were formed by raytracing methods. Images were reconstructed with the image pixel size equal to the detector pixel size $(3.3 \mathrm{~mm})$ and half of the detector pixel size $(1.65 \mathrm{~mm})$. The acceptance angle was 10 degrees. Counts were allocated to image pixels intersected by LORs. Contrast values were computed for the simulated tumors using the expression

$$
C=(T-B) / B
$$

where $T$ is the mean in a region of interest (ROI) including the tumor and $B$ is the mean in an ROI in the background region. The ROIs were $2 \times 2$ pixels in size.

Images of the compressed breast for the experimental acquisition described in section II.A were reconstructed using $3.3 \mathrm{~mm}$ and $1.65 \mathrm{~mm}$ pixels. There was no limit on the acceptance angle. Contrast values and signal-to-noise ratios of the tumors were computed.

\section{Count Allocation}

In PEM image reconstruction by the backprojection method, LORs are usually traced between the centers of the appropriate detector pixels and counts in an image plane are allocated to the image pixel through which the LOR passes. Event detection can also be modeled by viewing coincidence 
events as occurring within the tube of response (TOR) connecting two detector pixels (Figure 3). The intersections of the TORs with the image planes are squares and counts are allocated to image pixels in proportion to the area of these squares that they overlay.

This TOR method of count allocation was tested for the numerical simulation described in section $I I . B$ and for the previously described experimental breast phantom acquisition. The pixel size was $1.65 \mathrm{~mm}$ for all reconstructions. Tumor contrast and signal-to-noise values were computed. ---

\section{RESULTS}

\section{A. Image Uniformity}

There is good qualitative agreement between the measured and simulated flood measurements (Figure 4a, 4b). The ratios of experimental to simulated floods are uniform except near the edge of the field of view (Figures 4c, 5). The lower values near the edge of the detectors may be due to lower detector sensitivity in these edge regions. The amplitudes of horizontal profiles through these images are within $6 \%$ of unity except near the edge of the field of view (Figure 5). A calculated uniformity correction using equation (1) may avoid the need for excessive flood measurements in different image planes.

The calculated uniformity correction is quite effective for the experimental compressed breast phantom acquisition (Figure 6). The acceptance angle can be increased while maintaining uniformity over the field of view. The number of coincidence events contributing to the reconstructed images was 9977 events (5 degrees), 35207 events (10 degrees), 98491 events (20 degrees) and 119139 events (no angular limit). A greater number of events contributing to an inage decreases the statistical noise in the image. The signal-tonoise ratios for the 3 largest tumors increase as the acceptance angle increases (Table I).

\section{B. Image Sampling}

The contrast of smaller tumors improves slightly with the smaller pixel size for breast phantom simulations (Figure 7, Table II).

There is also a small increase in contrast in the experimental acquisition. For example, the $9 \mathrm{~mm}$ tumor in the upper right is more easily seen with finer pixelization (Figure 8, Table III). The signal-to-noise ratio decreases, however, since fewer events are contributing to each reconstructed pixel.

\section{Count Allocation}

The tube of response overlay method of count allocation eliminates some banding artifacts that occur near the edge of the field of view and improves image uniformity for both the simulated and experimental breast phantom studies (Figures $9,10)$. This smoothing of the TOR count allocation method decreases contrast with respect to the LOR method for the smaller tumors (Tables IV, V) but increases the signal-tonoise ratio (Table V).
TABLE I

TUMor Signal-TO-NoISE RATIOS FOR A BREAST PHANTOM EXPERJMENT WITH DifFerent ACCEPTANCE ANGLES

\begin{tabular}{|c|c|c|c|c|}
\hline \multirow{2}{*}{$\begin{array}{l}\text { Tumor } \\
\text { Diameter }\end{array}$} & \multicolumn{4}{|c|}{ Acceptance Angle } \\
\hline & 5 degrees & 10 degers & 20 deques & No Limit \\
\hline $15 \mathrm{~mm}$ & 13.3 & 15.9 & 28.5 & 48.5 \\
\hline $12 \mathrm{~mm}$ & 11.3 & 12.0 & 22.7 & 37.9 \\
\hline $9 \mathrm{~mm}$ & 4.0 & 5.0 & 7.9 & 13.6 \\
\hline
\end{tabular}

Tumor Contrast for Breast Phantom Simulations and Different Image

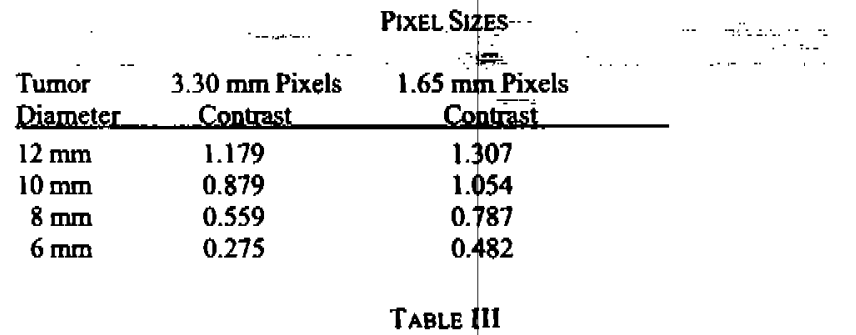

TUMor CONTRAST AND SIGNal-TO-NOISE RATIOS FOR A BREAST PHANTOM EXPERIMENT ANd Different Image PdXel SIZES

\begin{tabular}{lcccc} 
Tumor & \multicolumn{2}{c}{$3.30 \mathrm{~mm}$ Pixels } & \multicolumn{2}{c}{$1.65 \mathrm{~mm}$ Pixels } \\
Diameter & Contrast & S/N Ratio & Contrast & S/N Ratio \\
$15 \mathrm{~mm}$ & 2.06 & 48.5 & 2.20 & 24.2 \\
$12 \mathrm{~mm}$ & 1.61 & 37.9 & 1.92 & 21.1 \\
$9 \mathrm{~mm}$ & 0.58 & 13.6 & 0.71 & 7.8 \\
& & & \\
& & & TABLE IV
\end{tabular}

Tumor Contrast for Breast Phantom Smulations and Different Count Allocation Methods (1.65 mm Image Pixels)

$\begin{array}{llc}\begin{array}{c}\text { Tumor } \\ \text { Diameter }\end{array} & \begin{array}{c}\text { LOR Method } \\ \text { Contrast }\end{array} & \begin{array}{c}\text { TOR Method } \\ \text { Contrast }\end{array} \\ 12 \mathrm{~mm} & 1.307 & 1.266 \\ 10 \mathrm{~mm} & 1.054 & 0.997 \\ 8 \mathrm{~mm} & 0.787 & 0.706 \\ 6 \mathrm{~mm} & 0.482 & 0.403 \\ & & \\ & & \text { TABLE V }\end{array}$

Tumor Contrast and Signal-to-Noise Ratios for a BReast Phantom EXPERIMENT AND DifFerent CoUNT ALlocatTon METHODS (1.65 MM IMAGE PIXFis)

\begin{tabular}{lcccc} 
Tumor & \multicolumn{2}{c}{ LOR Method } & \multicolumn{2}{c}{ TOR Method } \\
Diameter & Contrast & SNRatio & Contrast & SN Ratio \\
\hline $15 \mathrm{~mm}$ & 2.20 & 24.2 & 2.21 & 42.9 \\
$12 \mathrm{~mm}$ & 1.92 & 21.1 & 1.83 & 35.6 \\
$9 \mathrm{~mm}$ & 0.71 & 7.8 & 0.67 & 13.1 \\
& \multicolumn{4}{c}{ IV. DISCUSSION }
\end{tabular}

Various factors affecting PEM image reconstruction have been investigated in this paper. The analytic image uniformity correction of equation (1) has been shown to be useful for application to an experimental acquisition, and we have also found it to be beneficial for all other PEM datasets to which it has been applied. Its practical application in this contribution assumes that the detector sensitivity functions are uniform. A more exact implementation would include compensations for nonuniformities in these intrinsic detector sensitivity functions.

There are tradeoffs between contrast and the signal-tonoise ratio in the choice of pixel size in the reconstructed images and in the choice of count allocation method. Image reconstruction with pixels half the size of the detector pixels and with the tube of response method seems to be a good 
compromise. Its contrast is almost as good as that achieved with the LOR method and with significantly better signal-tonoise ratios. Its contrast is better than that achieved with the image pixel size equal to the detector pixel size, and with very nearly the same signal-to-noise ratio. It is likely that an ROC study would be required to establish the optimal parameter choices for a given detection or quantitation task.

\section{CONCLUSION}

Three important factors affecting PEM - image reconstruction have been investigated-Analytic nodels for image uniformity can successfully model uniformity corrections as a function of acceptance angle for coincidence events. These analytic corrections can be successfully applied during image reconstruction and reduce the necessity for flood scans at different planes between the detectors. Smaller pixel sizes in image reconstruction increase the contrast of small lesions at the expense of reduced signal-to-noise ratios. A tube of response count allocation method greatly improves the signal-to-noise ratio at the expense of a slight decrease in image contrast, when compared with the more common line of response count allocation method. Further work is required to optimize image reconstruction parameters for particular detection or quantitation tasks.

\section{REFERENCES}

[1] R. L. Wahl, R. L. Cody, G. D. Hutchins, and e. al., "Primary and metastatic breast carcinoma: initial clinical evaluation with PET with the radiolabeled glucose analogue '2-[F-18]-fluoro-2-deoxy-D-glucose," Radiology, vol. 179, pp. $765+770,1991$.

[2] L. P. Adler, J. P. Crowe, N. K. Al-Kaisi, and J. L. Sunshine, "Evaluation of breast masses and axillary lymph nodes with [F-18] 2deoxy-2-fluoro-D-glucose PET," Radiology, vol. 187, pp. 743-750, 1993.

[3] N. Avril, M. Schelling, J. Dose, W. A. Weber, and M. Schwaiger, "Utility of PET in breast cancer," Clin. Pos. Imag., vol. 2, pp. 261-271, 1999.

[4] C. S. Yap, M. A. Seltzer, C. Schiepers, S. S. Gambhir, J. Rao, M. E. Phelps, P. E. Valk, and J. Czemin, "Impact of whole-body ${ }^{18}$ F-FDG PET on staging and managing patients with breast cancer: the referring physician's perspective," J. Nucl. Med, vol. 42, pp. 1334-1337, 2001.

[5] K. Murthy, M. Aznar, C. J. Thompson, A. Loutfi, R. Lisbona, and J. H. Gagnon, "Results of preliminary clinical trials of the positron emission mammography system PEM-I: a dedicated breast imaging system producing ghucose metabolic images using FDG," $J$. Nucl. Med., vol. 41, pp. 1851$1858,2000$.

[6] L. P. Adler, R. I. Freimanis, N. M. Lesko, S. Bergman, K, R Geisinger, E. A. Levine, N. Perrier, D. P. Newman, C. S. Sharpe, 'R. C. Williams, V. Zawarzin, and I. N. Weinberg, "Initial clinical results for FDG breast imaging with a dedicated PET camera," $J$. Nucl. Med., vol. 42, pp. 81P, 2001.

[7] C. J. Thompson, K. Murthy, I. N. Weinberg, and F. Mako, "Feasibility study for positron emission mammography," Med. Phys., vol. 21, pp. 529 $538,1994$.

[8] W. W. Moses, T. F. Budinger, R. H. Huesman, and S. E. Derenzo, "PET camera designs for imaging breast cancer and axillary node involvement," J. Nucl. Med, vol. 36, pp. 69P, 1995.

[9] R. H. Huesman, G. J. Klein, W. W. Moses, J. Qi, B. W. Reurter, and P. R. G. Virador, "List-mode maximum-likelihood reconstruction applied to positron emission mammography (PEM) with irregular sampling," IEEE Trans. Med. Imaging, vol. 19, pp. 532-537, 2000.

[10] G. D. Hutchins and A. J. Simon, "Evaluation of protorype geometries for breast imaging with PET radiopharmaceuticals, ${ }^{\prime} J$. Nucl. Med., vol. 36, pp. 69P-70P, 1995.

[11] I. N. Weinberg, S. Majewski, A. G. Weisenberger, A. Markowitz, L. Aloj, L. Majewski, D. Danforth, J. Mulshine, K. Cowan, J. Zujewski, C. Chow, E. Jones, V. Chang, W. Berg, and J. Frank, "Preliminary results for positron emission mammography: real-time functional breast imaging in a conventional mammographic gantry," Eur. J. Nucl. Med., vol. 23, pp. 804$806,1996$.

[12] R. Freifelder and J. S. Karp, "Dedicated PET scanners for breast imaging," Phys. Med. Biol., vol. 42, pp. 2463-2480, 1997.

[13] R. Freifelder, J. S. Karp, S. Surti, and J. A. Wear, "A dedicated PET scanner for breast imaging using two curve-plate NaI(TI) detectors," $J$ Nucl. Med., vol. 39, pp. 171P, 1998.

[14] R. R. Raylman, S. Majewski, R. Wojcik, A. G. Weisenberger, B. Kross, V. Popov, and H. A. Bishop, "The potential role of positron emission mammography for detection of breast cancer. A phantom sudy," Med. Phys., vol. 27, pp. 1943-1954, 2000.

[15] R. R. Raylman, S. Majewski, A. G. Weisenberger, V. Popov, R Wojcik, B. Kross, J. S. Schreiman, and H. A. Bishop, "Positron emission mammography-guided breast biopsy," $J$. . Nucl. Med., vol. 42, pp. 960-966, -2001 .

[16] H. Baghaei, W.-H. Wong, J. Uribe, H. Li, N. Zhang, and Y. Wang, "Breast cancer imaging studies with a variable field of view PET camera," IEEE Trans. Nucl. Sei., vol. 47, pp. 1080-1084, 2000.

[17] N. K. Doshi, Y. Shao, R. W. Silverman, and S. R. Cherry, "Design and evaluation of an LSO PET detector for breast cancer imaging," Med. Phys., vol. 27, pp. 1535-1543, 2000.

[18] C. J. Thompson, K. Murthy, Y. Picard, I. N. Weinberg, and R. Mako, "Positron emission mammography (PEM): a promising technique for detecting breast cancer," IEEE Trans. Nucl. Sci., vol. 42, pp. 1012-1017, 1995.

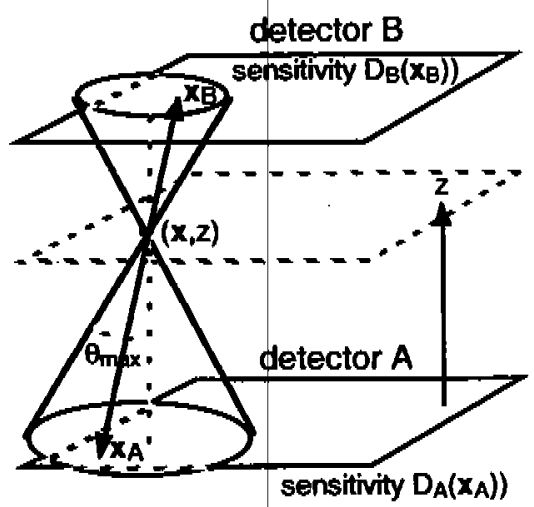

Fig. 1. Diagram of a point in plane $z$ between the two PEM detectors as part of a flood acquisition. Raypaths for a pair of annihilation photons are shown. Geometric sensitivity is reduced near the edge of the field of view.

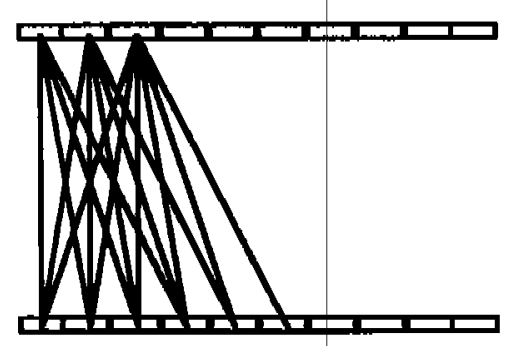

Fig. 2. Simplified diagram showing some of the lines of response between detector pixels for a dual detector PEM system. The raypath spacing in the plane midway between the detectors is half of the detector pixel spacing.

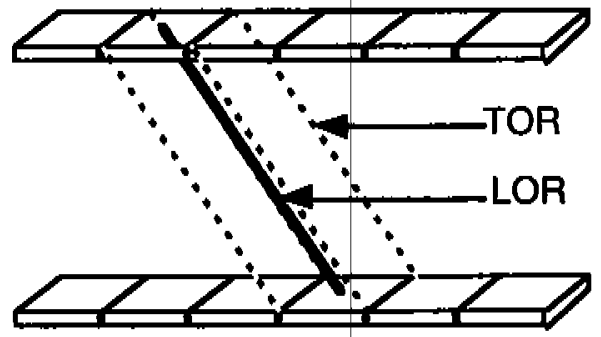

Fig. 3. Diagram showing the line of response (LOR) between two detector pixel centers (solid) and the boundaries of the tube of response (TOR) between the same detector pixels (dash). 


\section{A. Experiment}
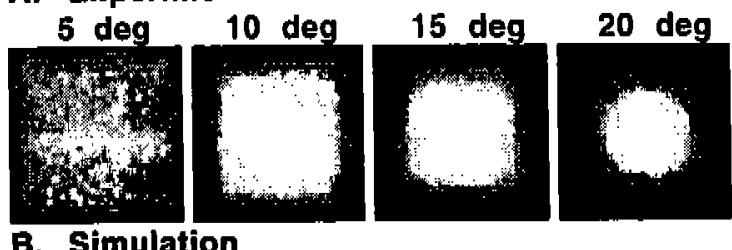

\section{B. Simulation}
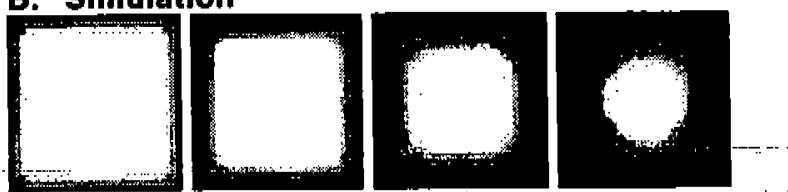

\section{Experiment:Simulation Ratio}
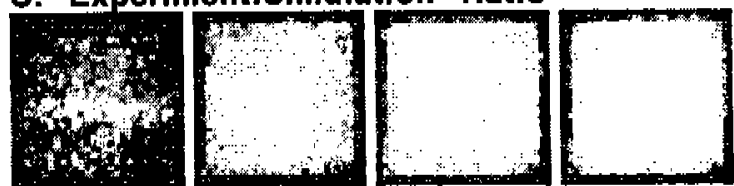

Fig. 4. (a) Experimental PEM flood images from a pixellated LGSO detector. The acceptance angle used in image reconstruction is indicated. (b) Simulated flood images and (c) ratio of experimental to simulated images.

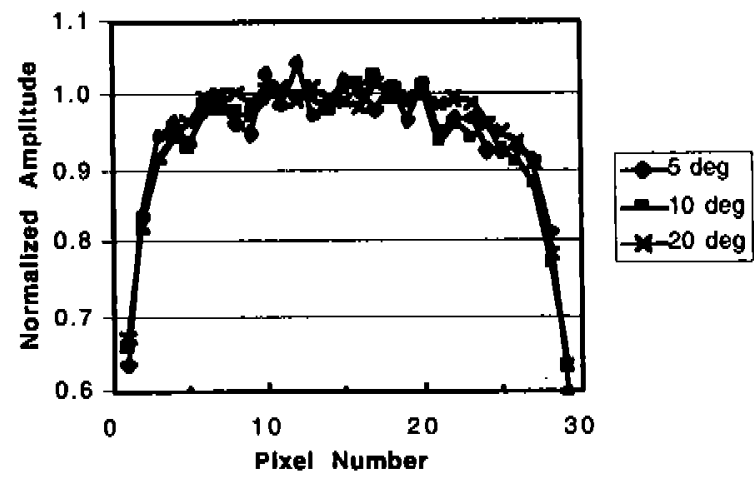

Fig. 5. A four pixel wide horizontal profile through the middle of the images of Figure $4 c$. The amplitudes are nommalized by the average of the center 10 pixels.

\section{A. No uniformity correction}

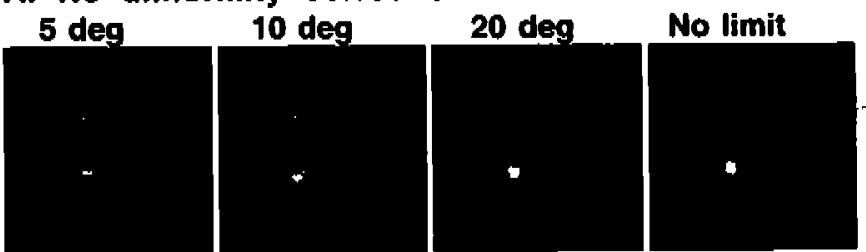

\section{B. Uniformity correction}

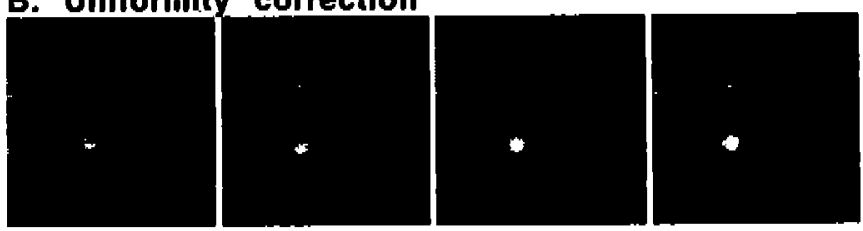

Fig. 6. Image reconstructions of an experimental compressed breast phantom acquisition for different acceptance angles (a) without and (b) with calculated uniformity corrections.

A. $3.3 \mathrm{~mm}$ pixels
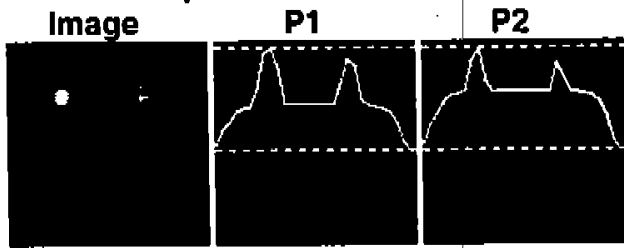

B. $1.65 \mathrm{~mm}$ pixels

Image
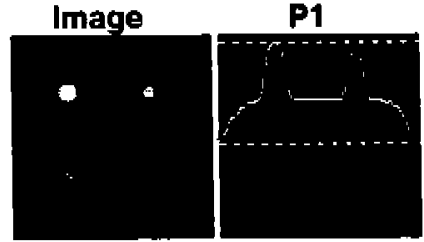

P2

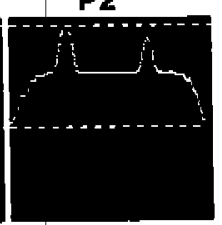

Fig. 7. Image reconstructions for the simulated compressed breast phantom acquisition with a 10 degree acceptance angle and (a) $3.3 \mathrm{~mm}$ image pixels, the same as the detector pixel size and (b) $1.65 \mathrm{~mm}$ image pixels, half of the size of the detector pixels. Profiles P1 are through the upper 12 and $10 \mathrm{~mm}$ diameter tumors, while the $P 2$ profiles are through the lower 8 and $6 \mathrm{~mm}$ diameter tumors.

\section{A. $3.3 \mathrm{~mm}$ pixels}

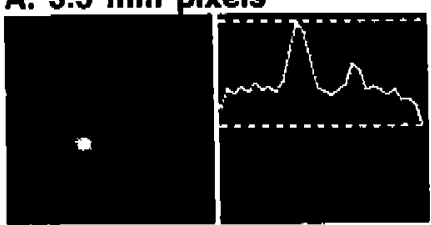

\section{B. $1.65 \mathrm{~mm}$ pixels}
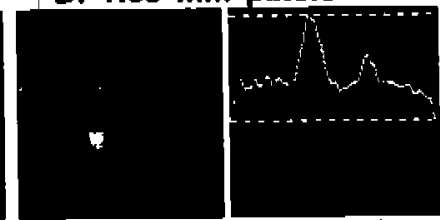

Fig. 8. Image reconstructions of an experimental compressed breast phantorn acquisition (a) with $3.3 \mathrm{~mm}$ image pixels, the same as the detector pixel size and (b) $1.65 \mathrm{~mm}$ image pixels, balf of the size of the detector pixels. The profiles are through the upper 12 and $9 \mathrm{~mm}$ diameter tumors. The acceptance angle was unlimited and a calculated uniformity correction was applied.
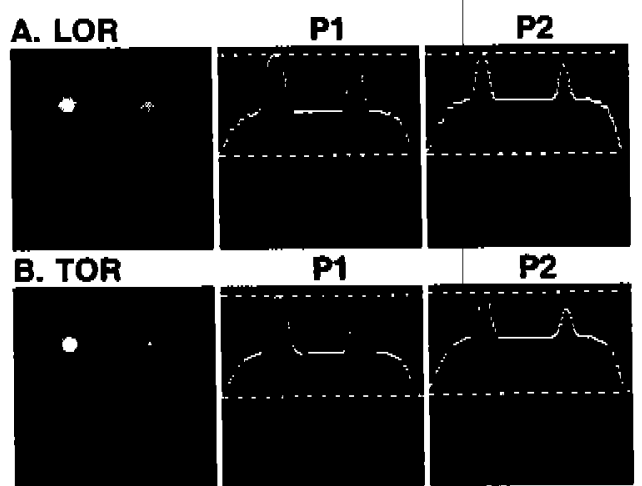

P1

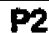

Fig. 9. Image reconstructions for the simulated compressed breast phantom acquisition with a 10 degre angular acceptance angle, $1.65 \mathrm{~mm}$ image pixels and (a) line of response (LOR) and (b) tube of response (TOR) count allocation methods. Profiles P1 are through the upper 12 and $10 \mathrm{~mm}$ diameter tumors, while the P2 profiles are through the lower 8 and $6 \mathrm{~mm}$ diameter tumors.

A. LOR

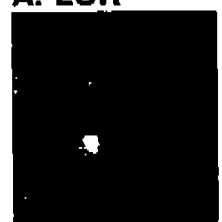

\section{B. TOR}

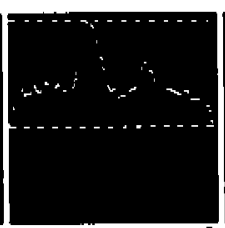

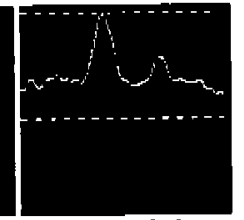

Fig. 10. Image reconstructions of an experimental compressed breast phantom acquisition with $1.65 \mathrm{~mm}$ image pixels and (a) line of response (LOR) and (b) tube of response (TOR) count allocation methods. The profiles are through the upper 12 and $9 \mathrm{~mm}$ diameter tumors. The acceptance angle was unlimited and a calculated uniformity correction was applied. 